to-face. The DNA rate was $11.2 \%$. In the three post-pandemic months this year, 101 clinics with 1171 patients were booked, $60.5 \%$ of these being telephonic, and total DNA was significantly reduced to $5.9 \%(\mathrm{p}<0.05)$. Additionally, there was a $38 \%$ increase in the numbers of patients assessed in the clinic. Conclusions The shift from having only face-to-face clinics to virtual clinics has led to increase in the number of clinics allowing more patients to be assessed, with improved DNA rates during and after the pandemic. Given the nature of the specialty, a large proportion of children will continue to need on-site assessment, but the service challenges imposed by the pandemic has created opportunities for smarter and higher volume working using technological means within a busy outpatient service.

\section{PAEDIATRIC SPINAL CORD INTRAMEDULLARY GLIOMAS SAFE MAXIMAL EXTENT OF RESECTION TO OPTIMIZE NEUROLOGICAL AND ONCOLOGICAL OUTCOMES}

Alexandra Valetopoulou, Maria Constantinides, Dulanka Silva, Dominic Thompson. Department of Neurosurgery, Great Ormond Street Hospital for Children NHS Foundation Trust

\subsection{6/archdischild-2020-gosh.114}

Objective Low grade intramedullary spinal cord tumours (IMSCTs) are rare tumours of childhood with potential for significant late morbidity following surgery.

We present our institutional experience with surgical treatment of these complex lesions emphasizing consistent definition of extent of resection (EOR), techniques to maximise surgical safety and a novel stratification of residual disease to guide post-operative strategy.

Methods A retrospective review of low-grade IMSCTs treated at GOSH between 2000 and 2019 was conducted. All surgery carried out by a single surgeon with intent of safe maximal resection guided by intra-operative neurophysiological monitoring (IONM). Pre and post-operative MRI were reviewed by neuro-radiologists.

EOR was recorded as:

1. Gross Total Resection (GTR) $-100 \%$ resection

2. Near Total Resection (NTR) - at least $95 \%$ resection

3. Sub Total Resection (STR) $-90 \%$ tumour resection

4. Partial Resection (PR) - less than 90\% tumour resection

Further outcome measures were time to recurrence, need for adjuvant therapy and mobility at last follow-up.

Results A total of 30 patients underwent surgery. IONM parameters (Motor evoked potentials, D-wave) were used to guide EOR. EORs achieved: GTR $=8, \mathrm{NTR}=4, \mathrm{STR}=9$, $\mathrm{PR}=9$.

All patients were alive at last follow up with eighteen patients (60\%) remaining radiologically and clinically stable. Twelve patients developed recurrence/progressive disease during surveillance (40\%) requiring adjuvant treatment. Progression free survival was significantly better in cases with GTR + NTR in comparison to either STR or PR.

Following surgery, 26/30 patients were independently mobile, $1 / 30$ required crutches and $3 / 30$ required a wheelchair.
9/30 patients were treated with adjuvant therapy following surgery.

Conclusion Survival rates for low grade IMSCT are excellent. Radical micro-surgical resection, guided by IONM provides an effective means of balancing the objectives of maximal safe resection, functional outcome and tumour control. Small volume residual disease does not compromise long-term oncological outcome.

\section{FAMILY EXPERIENCES OF FLYING AND TESTING: 'FITNESS TO FLY'}

${ }^{1}$ Katharine Pike, ${ }^{2}$ Paula Kelly, ${ }^{3}$ Stephanie Brotherston, ${ }^{3}$ Mollie Riley, ${ }^{4}$ Martin Samuels. ${ }^{1}$ Bristol Royal Hospital for Children; ${ }^{2}$ ORCHID -BRC NIHR Great Ormond Street Hospital for Children NHS Foundation Trust; ${ }^{3}$ Great Ormond Street Hospital for Children NHS Foundation Trust; ${ }^{4}$ Respiratory Medicine Great Ormond Street Hospital for Children NHS Foundation Trust

\subsection{6/archdischild-2020-gosh.115}

As part of a feasibility study investigating a new protocol for hypoxic challenge testing (HCT) in children with neuromuscular weakness or central hypoventilation who use ventilation at home, we conducted interviews evaluating parents' and children's experiences of flying and this pre-flight testing. The new protocol may better advise patients who use ventilators about their 'fitness to fly'.

We have recruited 15 children, 7 with neuromuscular weakness, and 8 with central hypoventilation to date (mean age 8.5 years, range 1.7 to 16.7 years), all of whom have sat in an airtight chamber with an oxygen concentration 15\% (normal $21 \%$ ) to simulate the in-flight environment. Patients had monitoring of oxygen saturation, end tidal and transcutaneous carbon dioxide in air, 15\% oxygen, and then with supplemental oxygen, ventilation and if needed, both.

Semi-structured interviews immediately after the HCT and by telephone 3-4 months later identified the following factors from the preliminary analysis of interview transcripts.

Parents identified the challenges they faced when planning and undertaking air-travel, including repeated negotiations with airlines, on provision of oxygen, accommodating essential equipment thereby requiring families to be proactive managers. Despite these challenges several families were frequent travellers, who valued opportunities to visit extended family and enjoy new experiences as a whole family. During interviews older children identified travel as an opportunity for normalisation of their lives. Some families remained cautious about air travel expressing concerns about their child's underlying vulnerability.

Parents and children provided valuable feedback on the experience of the HCT protocol, including challenges of space for wheelchairs and equipment in the chamber, the importance of considering school attendance in the context of multiple hospital appointments.

Overall the testing process and results were seen positively by many, it built confidence for parents by providing more information about their child's responses to flight to aid future decision making. 